\title{
MAD FAMILIES AND ULTRAFILTERS
}

\author{
MARTIN WEESE ${ }^{1}$
}

\begin{abstract}
For each almost disjoint family $X$ let $F(X)=\{a \subseteq \omega$ : card $\{s \in X$ : $s \backslash a$ is finite $\left.\}=2^{\omega}\right\}, I(X)=\left\{a \subseteq \omega:\right.$ card $\{s \in X:$ card $\left.(s \cap a)=\omega\}=2^{\omega}\right\}$. Assuming $P\left(2^{\omega}\right)$ we show that for each nonprincipal ultrafilter $p$ there exist a maximal almost disjoint family $X$ and an almost disjoint family $Y$ with $F(X)=$ $I(Y)=p$.
\end{abstract}

1. Introduction. We refer the reader to [2] for unexplained notions. Let $A$ be a set; $\mathcal{P}(A)$ denotes the power set of $A$ and card $A$ denotes the cardinality of $A$. Fin denotes the set of finite subsets of $\omega$. For $a, b \in \mathscr{P}(A)$ we write $a \subseteq{ }_{*} b$ if $a \backslash b$ is finite and we write $a={ }_{*} b$ if $a \subseteq{ }_{*} b$ and $b \subseteq{ }_{*} a$.

Let $X \subseteq \mathscr{P}(\omega) \backslash$ Fin. $X$ has the fip (finite intersection property) if for any finite subset $S$ of $X, \cap S$ is infinite. $X$ is almost disjoint if (i) for $a, b \in X$ with $a \neq b$, $a \cap b \in$ Fin and (ii) for any finite subset $S$ of $X, \omega \backslash \cup S$ is infinite. $X$ is called mad family if it is a maximal almost disjoint family and $X$ is called ad family if it is an almost disjoint family.

Let $P\left(2^{\omega}\right)$ be the following proposition (considered by Rothberger [5]):

If $F \subseteq \mathscr{P}(\omega)$ has the fip and card $F<2^{\omega}$ then there is $d \in \mathscr{P}(\omega) \backslash$ Fin with $a \subseteq{ }_{*} b$ for each $b \in F$.

The proposition $P\left(2^{\omega}\right)$ is weaker than Martin's axiom (see [4]).

For $X$ an ad family we set

$$
\begin{aligned}
& F(X)=\left\{a \subseteq \omega: \operatorname{card}\left\{s \in X: s \subseteq{ }_{*} a\right\}=2^{\omega}\right\} \\
& I(X)=\left\{a \subseteq \omega: \operatorname{card}\{s \in X: \operatorname{card}(s \cap a)=\omega\}=2^{\omega}\right\}
\end{aligned}
$$

Then for each ad family $X, F(X) \subseteq I(X)$; for $X$ a mad family, $I(X)=\{a \subseteq \omega$ : for each finite subset $S$ of $X, \operatorname{card}(a \backslash \cup S)=\omega$. We show:

THEOREM 1. Assume $P\left(2^{\omega}\right)$. Then for any nonprincipal ultrafilter $p$ on $\omega$ there exists a mad family $X$ with $F(X)=p$.

THEOREM 2. Assume $P\left(2^{\omega}\right)$. Then for any nonprincipal ultrafilter $p$ on $\omega$ there exists an ad family $X$ with $I(X)=p$.

Received by the editors August 11, 1978 and, in revised form, July 16, 1979.

AMS (MOS) subject classifications (1970). Primary 02K05; Secondary 06A40, 54A25, 54D35.

Key words and phrases. Almost disjoint family, Stone-Cech compactification, $2^{\omega}$-point, superatomic Boolean algebra.

${ }^{1}$ The author wishes to thank the referee and J. E. Baumgartner for many valuable remarks. 
2. Proof of Theorems 1 and 2. Let $p$ be any nonprincipal ultrafilter on $\omega$, let $\left\{a_{i}: i<2^{\omega}\right\}$ be an enumeration of $p$ such that for each $b \in p$ we have $\operatorname{card}\left\{i<2^{\omega}: b=a_{i}\right\}=2^{\omega}$ and let $\left\{b_{i}: i<2^{\omega}\right\}$ be an enumeration of $\{b \subseteq \omega$ : $b \notin p$, card $b=\omega\}$. Let $A_{k}=\left\{a_{i}: i<k\right\}, B_{k}=\left\{b_{i}: i<k\right\}$. We construct increasing sequences $\left\{X_{i}: i<2^{\omega}\right\},\left\{Y_{i}: i<2^{\omega}\right\}$ of almost disjoint sets such that for each $i<2^{\omega}$ :

(i) card $X_{i}<2^{\omega}$ and card $Y_{i}<2^{\omega}$;

(ii) $\left(X_{i} \cup Y_{i}\right) \cap p=\varnothing$;

(iii) $X_{i} \cap Y_{i}=\varnothing$;

(iv) there is $c \in X_{i+1} \backslash X_{i}$ with $c \subseteq a_{i}$;

(v) there is $d \in Y_{i+1}$ with $\operatorname{card}\left(d \cap b_{i}\right)=\omega$;

(vi) for $i<k<2^{\omega}$, if $c \in X_{k} \backslash X_{i}$, then $\operatorname{card}\left(c \cap b_{i}\right)<\omega$;

(vii) for $i<k<2^{\omega}$, if $d \in Y_{k} \backslash Y_{i}$, then $\operatorname{card}\left(d \cap b_{i}\right)=\omega$.

Let $X=\cup\left\{X_{i}: i<2^{\omega}\right\}, Y=\cup\left\{Y_{i}: i<2^{\omega}\right\}$. Then $X$ is an ad family and (v) implies that $X \cup Y$ is a mad family. (iv) implies that for each $a \in p, a \in F(X)$ and $a \in F(X \cup Y)$. (vi) implies that for each $a \subseteq \omega$ with $a \notin p, a \notin I(X)$. (vii) implies that for each $a \subseteq \omega$ with $a \notin p, a \notin F(X \cup Y)$. Thus $I(X)=F(X \cup Y)=p$.

Now we describe the construction of the $X_{i}$ and $Y_{i}$. We set $X_{0}=Y_{0}=\varnothing$. Assume $i<2^{\omega}$ and for each $k<i, X_{k}$ and $Y_{k}$ are constructed. For $i$ a limit ordinal we set $X_{i}=\bigcup\left\{X_{k}: k<i\right\}, Y_{i}=\bigcup\left\{Y_{k}: k<i\right\}$.

Now let $i$ be a successor ordinal, $i=k+1$. Let $S=A_{i} \cup\left\{\omega \backslash b: b \in B_{i}\right\} \cup$ $\left\{\omega \backslash x: x \in X_{k}\right\}$. Then $S$ has the fip and card $S<2^{\omega} . P\left(2^{\omega}\right)$ implies that there is $a \subseteq \omega$ with $a \backslash s \in$ Fin for each $s \in S$. Let $a^{*} \subseteq a \cap a_{i}$ be such that $a^{*} \notin p$ and card $a^{*}=\omega$. Then we set $X_{i}=X_{k} \cup\left\{a^{*}\right\}$. Assume there is $s \in X_{i} \cup Y_{k}$ with $\operatorname{card}\left(s \cap b_{i}\right)=\omega$. Then we set $Y_{i}=Y_{k}$. Assume now that no such $s$ exists. Let $T=A_{i} \cup\left\{\omega \backslash b: b \in B_{i}\right\} \cup\left\{\omega \backslash x: x \in X_{i}\right\}$. Then $T$ has the fip and card $T<2^{\omega}$. $P\left(2^{\omega}\right)$ implies that there is $c \subseteq \omega$ with $c \backslash s \in$ Fin for each $s \in T$. Let $c^{*} \subseteq c \cap a_{i}$ be such that $c^{*} \notin p$ and card $c^{*}=\omega$. Then we set $Y_{i}=Y_{k} \cup\left\{c^{*} \cup b_{i}\right\}$. It is now easy to see that (i)-(vii) are satisfied.

3. Topological consequences. Let $N$ be the discrete countable space and let $\beta N$ be the Stone-Čech compactification of $N$. Then $\beta N \backslash N$ can be represented by the set of all nonprincipal ultrafilters over $\omega$ and the topology generated by the following basis $\mathfrak{A}$ : For each $a \subseteq \omega$ let $\hat{a}=\{p \in \beta N \backslash N: a \in p\}$ and $\mathfrak{A}=\{\hat{a}: a \subseteq \omega\}$. Then $\hat{a} \supseteq \hat{b}$ iff $b \subseteq{ }_{*} a$. Then Theorems 1 and 2 can be reformulated as follows:

TheOREM $1^{\prime}$. Assume $P\left(2^{\omega}\right)$. Then for each $p \in \beta N \backslash N$ there is a dense system $\mathfrak{u}_{p}$ of open sets such that for each $a \subseteq \omega, a \in p$ iff $\operatorname{card}\left\{U \in \mathfrak{U}_{p}: U \subseteq \hat{a}\right\}=2^{\omega}$.

TheOREM $2^{\prime}$. Assume $P\left(2^{\omega}\right)$. Then for each $p \in \beta N \backslash N$ there is a system $\mathfrak{u}_{p}$ of open sets such that for each $a \subseteq \omega, a \in p$ iff $\operatorname{card}\left\{U \in \mathfrak{U}_{p}: U \cap \hat{a} \neq \varnothing\right\}=2^{\omega}$.

$p \in \beta N \backslash N$ is a $2^{\omega}$-point if there is a family $\left\{U_{i}: i<2^{\omega}\right\}$ of pairwise disjoint open sets with $p \in\left(\operatorname{cl}_{\beta N} U_{i}\right) \backslash N$. We can use Theorem 1 to derive the following theorem of Hindman [3] (Hindman used $\mathrm{CH}$ but there is little difficulty adapting his proof to $\left.P\left(2^{\omega}\right)\right)$ : 
TheOREM 3. Assume $P\left(2^{\omega}\right)$. Then each $p \in \beta N \backslash N$ is a $2^{\omega}$-point.

Proof. Let $X=\left\{c_{i}: i<2^{\omega}\right\}$ be a mad family with $F(X)=p$. For each $i<2^{\omega}$ choose an ad family $\left\{d_{i k}: k<2^{\omega}\right\}$ with $d_{i k} \subseteq c_{i}$ for each $k<2^{\omega}$. For $k<2^{\omega}$ let

$$
U_{k}=\cup\left\{\hat{d}_{i k}: i<2^{\omega}\right\} \text {. }
$$

Then the $U_{k}$ are pairwise disjoint open sets and $p$ is in the closure of each $U_{k}$.

REMARK. Balcar and Vojtáš [1] proved Theorem 3 without any set-theoretical assumption. It is also unknown whether Theorem 1 holds without any set-theoretical assumption.

4. Applications to superatomic Boolean algebras. Let $\mathfrak{A}$ be a Boolean algebra. $a \in|\mathfrak{A}|$ is an atom if $a \neq 0$ and for each $b \in|\mathfrak{U}|, a \cap b=a$ or $a \cap b=0 . \mathfrak{U}$ is atomic if for each $b \in|\mathfrak{A}|$ there is an atom $a$ with $a<b$. $\mathfrak{A}$ is superatomic if each homomorphic image of $\mathfrak{A}$ is atomic. $\underline{2}$ denotes the two-element Boolean algebra, $\operatorname{Pow}(\omega)$ denotes the power set Boolean algebra over $\omega$. For $A \subseteq \operatorname{Pow}(\omega)$ let $\operatorname{Pow}(\omega)[A]$ denote the subalgebra of $\operatorname{Pow}(\omega)$ generated by $A \cup \omega$. For each Boolean algebra $\mathfrak{A}, \mathfrak{A}^{(1)}$ denotes $\mathfrak{A}$ factorized by the ideal generated by the atoms and for each $k \in \omega$ we set $\mathfrak{A}^{(k+1)}=\left(\mathfrak{U}^{(k)}\right)^{(1)}$. If $X$ is a mad family then $\operatorname{Pow}(\omega)[X]$ is a superatomic Boolean algebra whose set of atoms is $\omega$ and $(\operatorname{Pow}(\omega)[X])^{(2)} \cong \underline{2}$.

TheORem 4. Assume $P\left(2^{\omega}\right)$. Then there are $2^{2^{\omega}}$ nonisomorphic superatomic Boolean algebras $\mathfrak{A}$ whose set of atoms is $\omega$ and with $\mathfrak{A}^{(2)} \simeq \underline{2}$.

Proof. Let $\mathfrak{X}$ be the class of all mad families $X$ such that $F(X)$ is a nonprincipal ultrafilter. Let $X, Y \in \mathfrak{X} . X$ and $Y$ ar called equivalent if there are $a \in X, b \in Y$ and a one-one function $f$ from a onto $b$ such that for each $s \in X$ with $s \subseteq{ }_{*} a$ there is $t \in Y$ with $f[s]={ }_{*} t$. That means, $X$ and $Y$ are equivalent iff $F(X)$ and $F(Y)$ are equivalent with respect to the Rudin-Keisler order of ultrafilters. Now there are $2^{2^{\omega}}$ nonprincipal ultrafilters on $\omega$ and each equivalence class with respect to the Rudin-Keisler order contains $2^{\omega}$ ultrafilters. Let $\mathfrak{\Im} \subseteq \mathfrak{X}$ be such that card $\mathfrak{\Im}=2^{2^{\omega}}$ and the elements of $\mathfrak{\Im}$ are pairwise nonequivalent. Let

$$
\Re=\{\operatorname{Pow}(\omega)[X]: X \in \mathfrak{s}\} .
$$

Then $\Re$ is the desired class of superatomic Boolean algebras.

ADDED IN PROOF. As I was informed by Baumgartner, it is impossible to prove Theorem 1 without any set-theoretical assumption.

\section{REFERENCES}

1. B. Balcar and P. Vojtá, Almost disjoint refinement of families of subsets of $N$ (preprint).

2. W. Comfort and S. Negrepontis, The theory of ultrafilters, Springer-Verlag, Berlin and New York, 1974.

3. N. Hindman, On the existence of $\tau$-points in $\beta N \backslash N$, Proc. Amer. Math. Soc. 21 (1969), 277-280.

4. K. Kunen and F. D. Tall, Between Martin's axiom and Souslin's hypothesis, Fund. Math. 102 (1979), 173-181.

5. F. Rothberger, On some problems of Hausdorff and of Sierpinski, Fund. Math. 35 (1948), 29-46.

Humboldt-Universität zu Berlin, Unter den Linden 6, 108 Berlin, German Democratic REPUBLIC 